\title{
TEMPORARY HENNA TATTOOS - NEW ASPECTS OF ALLERGY TO PARA-PHENYLENE DIAMINE
}

\author{
Jana Kazandjieva, Nikolai Tsankov \\ Department of Dermatology, \\ Medical University - Sofia, Bulgaria
}

\section{SUMMARY:}

Para-phenylene diamine is a powerful and well known allergen. Nevertheless PPD is widely used for the making of temporary henna tattoos. It is added to the henna paste and afterwards applied directly onto the skin. This way the popular henna tattoos may cause allergic reactions. We present two patients with allergic reactions due to temporary henna tattoos. Patch testing showed a strongly positive reaction to paraphenylene diamine. People with known reactions to PPD or cross-reacting allergens such as sulfonamides, para-amino benzoic acid, sulfonylureas, dapsone, azo dyes or benzocaine should be especially cautious of black henna tattoo application.

Key words: contact dermatitis, henna, para-phenylene diamine, temporary henna tattoos

\section{INTRODUCTION}

Paraphenylenediamine dihydrochloride (PPD) also called 1,4-diaminobenzene or 1,4-phenylenediamine is an aromatic amine. It is the main component of all black permanent hair dyes available on the market, no matter the trade name. By default the darker the hair day is the higher concentration of PPD it has. Even the so called "natural" hair dyes contain PPD.

Because PPD is a powerful and well known allergen its use is allowed in the EU for hair dyes in a maximum concentration of $6 \%$ free base in the final product(1). The same directive forbids the use of PPD for dying lashes, eyebrows, or skin. Nevertheless PPD is widely used for the creation of temporary henna tattoos. It is added to the henna paste and afterwards applied directly onto the skin. That way PPD assures the darkening of the color (from orange to black) and the prolonged lasting of the skin painting (from 7 to 20 days)

According to the literature the skin is the major target of allergic reactions to PPD. That's why we recently observed many cases of allergic reactions to temporary henna tattoos.

We present 2 cases of allergic contact dermatitis after temporary "henna" tattoos.

\section{CASE REPORTS}

Case 1:

13 year old girl made her first henna tattoo in 2003. At the time she noticed slight erythema and pruritus on the tattooed place, but they quickly faded away. In 2004 the patient had made another temporary henna tattoo and a severe bullous reaction was present on the site of the tattoo.

Dermatological status: the pathological changes are localized on the back and are presented with a bullous reaction arising directly in the center of the tattoo. In the bullous lesion particles from the tattoo pigment can be observed. The surrounding skin is unchanged. /Fig. 1/. After a treatment with systemic antihistamines and local corticosteroids the pathological changes disappeared. One month later the patient was tested in our allergology lab and the epicutaneous tests was positive for PPD.

\section{Case 2:}

The patient is a 19 year old boy, who for the first time decided to tattoo himself with commercial henna (the so-called black henna). Three days later papulo-vesiculous skin lesions appeared on his arm, where the tattoo was painted.

Dermatological status: the skin lesions are localized on the left arm and are presented by an erythematosus plaque with severe exudation and impetiginisation /Fig.2/. Treatment with topical steroids (class IV) and antibiotics resulted in gradual improvement with resolution in several weeks.Again, the epicutaneous tests were positive for PPD and commercial henna.

\section{DISCUSSION :}

PPD is the leading hair coloring agent since its introduction in the $1880 \mathrm{~s}$. Because of its allergic potential it has been problematic almost from the start. PPD is a very common allergen among patients with allergic contact dermatitis. Surprisingly, in "Dermatitis" some authors proclaimed for contact allergen of the year 2006 - p-Phenylenediamine(2). According to them PPD was chosen because recently a number of changes have occurred in dealing with allergy to this antigen. Especially the recognition of new patterns of exposure to PPD - namely the temporary henna tattoos - was a main reason for this nomination.

The allergic dermatitis to PPD after the application of a temporary tattoo has its own specific etiology, incubation period, clinical picture and complications. Basing on our own cases and the documented ones in the literature we made our own clinical research concerning the main characteristics of this special clinical presentation(3). In our study patch testing 
showed $87 \%$ positive reactions to PPD, $6 \%$ to henna mix and $7 \%$ showed negative result. There were no positive reactions to natural henna. Therefore PPD added to the henna paste provoked the allergic reaction. In our study $82 \%$ of the patients were with the typical clinical picture of acute allergic contact dermatitis. In $18 \%$ of the cases the clinical findings were with the characteristics of lichenoid contact dermatitis. Another important observation was the long lasting hyperor hypo pigmentation (in $86 \%$ of the cases more then 6 months).

Recently in "Contact Dermatitis"(4) a study demonstrated for the first time that, over the time period tested, the allergenic component of PPD accumulates in the skin. Hence, intermittent exposure to lower concentrations of PPD may be equivalent to a higher concentration in a one-off exposure. This statement is of main importance for the temporary tattoos. In one hand high concentration of PPD in the henna paste may lead to sensitization just after the first "henna painting". On the other hand the fashion to be tattooed every summer assured an intermittent exposure to low concentra- tions of PPD and it may also lead to sensitization due to the accumulative effect in the skin.

Sensitization is a lifelong issue. PPD cross-reacts with related compounds that have an amino group in the c-position of the benzene ring. People with known reactions to PPD or cross-reacting allergens such as sulfonamides, c -amino benzoic acid, sulfonylureas, dapsone, azo dyes or benzocaine should be especially cautious of black henna tattoo application(5). Sensitization and cross-reactivity are not limited to the specific chemicals themselves. Allergic patients to PPD should avoid hair coloring, printer ink, photographic products and black clothing dyes. To top that, there are reports concerning a possible association between PPD exposure and the increased risk of developing bladder cancer in humans and rats. It is suggested that this may be due to the slower rate of PPD detoxification, resulting in metabolite formations(6).

It is clear that PPD - a new old allergen has different masks and is of great importance for our own health to recognize them all.

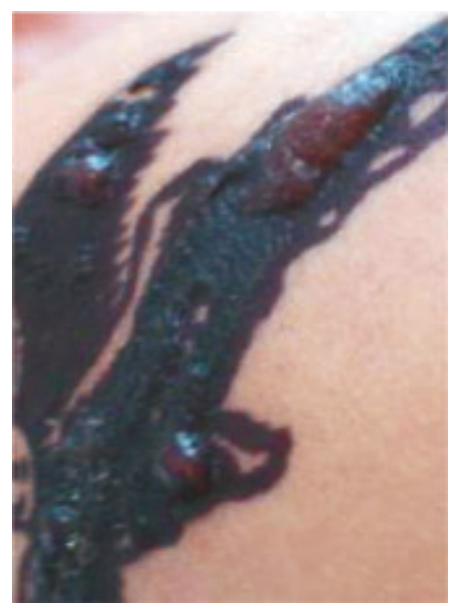

Fig. 1 - Case 1 - bullous reaction on the site of the tattoo

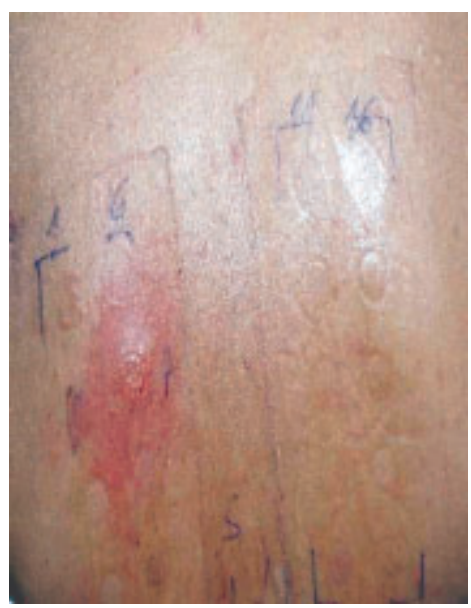

Fig. 2 - Case 1 - positive epicutaneous test to PPD

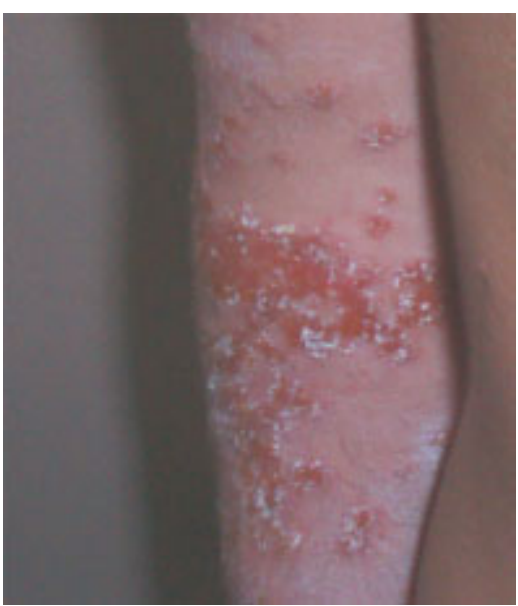

Fig. 3 - Case 2 - allergic contact dermatitis resulting from a temporary tattoo

\section{REFERENCES:}

1. Brancaccio RR, Brown LH, Chang YT, Fogelman JP, Mafong EA, Cohen DE. Identification and quantification of paraphenylenediamine in a temporary black henna tattoo. Am J Contact Dermat 2002,13,15-8

2. DeLeo VA. Contact Allergen of the Year: p-Phenylenediamine. Dermatitis, Posted 10/24/2006
3. Kazandjieva J, Grozdev I, Tsankov N. Temporary henna tattoos. Clin in Dermatol, 2007, 25, 383-387

4. White J,. Basketter D, Pease C, Sanders D, McFadden J Intermittent exposure to low-concentration paraphenylenediamine can be equivalent to single, higher-dose exposure. Contact Dermatitis 200756 (5), 262-265
5. Arroyo M. Black henna tattoo reaction in a person with sulfonamide and benzocaine drug allergies. JAAD, 2003,48

6 . Nohynek GJ, et al. Urinary acetylated metabolites and $\mathrm{N}$-acetyltransferase-2 genotype in human subjects treated with a para-phenylenediamine-containing oxidative hair dye. Food Chem Toxicol. 2004 Nov; 42(11):1885-91

\section{Address for correspondence:}

Dr Jana Kazandjieva, Department of Dermatology, Medical University - Sofia, 1, St. George Sofiiski Str., 1431 Sofia, Bulgaria E-mail: janaderm@abv.bg; 